\title{
Distributed Robust Filtering over Sensor Networks with Quantized Measurement and Switching Topologies
}

\author{
Shuchen Ding and Fengzeng Zhu *ii \\ Engineering Research Center of Internet of Things Applied Technology, Jiangnan University, Wuxi 214122, China; \\ shuchendsc@jiangnan.edu.cn \\ * Correspondence: zhufengzeng@stu.jiangnan.edu.cn
}

\begin{abstract}
This paper aims at exploring the theoretical research and distributed filtering design of state estimation for sensor networked systems with quantized measurement and switching topologies. In a sensor network, each sensor node has an independent static logarithmic quantizer function, and the quantized measurement is transmitted to the filtering network via the wireless network. In the corresponding filtering network, each local estimator achieves distributed consistent state estimation of the plant based on the local measurement and the neighboring shared information. In particular, the design of the distributed filter fully takes into account the fact that the communication links between the nodes are not fixed. That is, the communication topology has random switching, and such random switching behavior is described using Markov chains with partially unknown transition probabilities. A set of linear matrix inequalities gives the sufficient conditions for the existence of the distributed filter, while ensuring that the filter error system has the desired $H_{\infty}$ performance. Finally, two numerical simulations show the effectiveness of the design method.
\end{abstract}

Keywords: wireless sensor network; distributed filtering; robust performance; unknown transition probability; free-connection weighting matrix

Distributed Robust Filtering over Sensor Networks with Quantized Measurement and Switching Topologies. Electronics 2021, 10, 2336. https://doi.org/10.3390/ electronics10192336

Academic Editor: Hamid Reza Karimi

Received: 1 September 2021

Accepted: 19 September 2021

Published: 23 September 2021

Publisher's Note: MDPI stays neutral with regard to jurisdictional claims in published maps and institutional affiliations.

Copyright: (c) 2021 by the authors. Licensee MDPI, Basel, Switzerland. This article is an open access article distributed under the terms and conditions of the Creative Commons Attribution (CC BY) license (https:// creativecommons.org/licenses/by/ $4.0 /)$.

\section{Introduction}

In recent years, wireless sensor networks (WSNs) have been widely used in various fields, such as environmental monitoring, smart grid, smart transportation, etc. [1-5]. Benefiting from the outstanding performance of micro-electro-mechanical systems (MEMS) technology, these sensors are compact, have limited processing and computing capabilities, and are relatively affordable in comparison to traditional sensors. Such sensor nodes are capable of sensing, measuring, and collecting data from their surroundings and transmitting it to the user based on a local decision-making process.

Simultaneously, more and more scholars are paying attention to the distributed filtering problem based on WSN. In a distributed filtering network, a filter node fuses local measurement and neighbor node information to achieve system state estimation. Compared with traditional centralized filtering, the distributed filtering is more robust in terms of sensor failures and transmission constraints, that is, when a node failure or data packet loss occurs, the filter node can still obtain information from other neighbor nodes, and the final estimation result will not be severely damaged. Among the existing results about distributed filters, the distributed Kalman filters [6-11] and distributed robust filters [12-20] are two important state estimators. For example, a distributed filtering algorithm based on Kalman consensus has been proposed in [7], which is suitable for sensor networks with time-varying transmission delays in communication links. In [9], a novel peer-to-peer distributed Kalman filter has been proposed, in which each sensor communicates with its connected neighbors to achieve the average consensus on weighted measurement and inverse covariance matrices. It should be pointed out that, for the distributed state estimator based on Kalman filter, accurate noise statistics is a necessary design prerequisite, and the distributed robust filter solves this shortcoming well. For example, in order to alleviate the 
burden of communication in WSNs, the literatures [12-14] have adopted a round-robin protocol to orchestrate the transmission order and addressed the problem of distributed set-member estimation. The literature [18] have developed a distributed event-triggered $H_{\infty}$ filtering algorithm, where the sensor saturation in the spatially distributed sensors and the randomly occurring cyber attacks are considered simultaneously.

The above-mentioned studies assumed a fixed filtering network topology. However,in many complex networks, network connectivity topologies might not be fixed, and might even randomly change over time (e.g., random failure and recovery of communication links, obstruction blocking, and uncertain network-induced phenomenon). To date, distributed filtering with switching topologies has achieved some results [21-23]. For example, a distributed $H_{\infty}$ state estimation approach has been proposed in [22,23] for discrete-time systems with random switching topologies, packet losses, and partial information exchange, where the switching topology is defined by non-homogeneous Markov chains. It is a pity that when a Markov chain is used to model switching topology, a complete state transition probability information must be obtained, which is a prerequisite for the existence of these research results. In fact, even for a simple system, it is quite difficult and expensive to accurately obtain the complete transition probability. Therefore, the development of a distributed $H_{\infty}$-consensus state estimation method with a time-varying switching topology subject to partially unknown transition probability is of great significance to achieve target estimation or tracking, which is the first motivation of this study.

On the other hand, the measured signal between the sensor network and the filtering network is transmitted through a limited capacity channel, and the signal needs to be quantized before transmission in many cases. However, signal quantization inevitably introduces quantization error, which affects system stability and robustness. Significant effort has been made to signal quantization for a variety of different sorts of systems [24-32]. In particular, in order to deal with the unboundedness of measurement information for unstable systems, the authors of [25] have transmitted the quantified innovation to the fusion center through a bandwidth-limited channel. Based on the bounded recursive optimization technology, the design of the local estimator and the distributed fusion estimator are transformed into two different convex optimization problems, which can be easily solved by standard software. In [28], an adaptive back-stepping control scheme for non-rigid spacecraft attitude tracking with input quantization, inertial uncertainty and external interference has been proposed, in which the control signal of each actuator is adopted logarithmic quantizer and hysteresis quantizer to perform signal quantization processing. The literature [31] introduces a novel non-stationary quantizer in the multi-level structure strategy to solve the non-stationary quantization control problem for a class of Markov switching systems, which involves randomly packet dropouts and a mode-dependent logarithmic quantizer. Given the fact that the WSN is composed of a large number of sensor nodes, the measurement error caused by quantization must be a non-negligible problem, which drives our second motivation for this research.

In this article, we focus on solving the distributed $H_{\infty}$ filtering problem for a class of discrete systems in a realistic sensor network and filtering network environment, while considering the effects of sensor quantization and switching topologies. The first contribution of this work is to embed a logarithmic signal quantizer for the sensor network, where the quantizers are static and independent of each other. The quantization error is constrained to a sector-bounded condition, which transforms the quantization problem into a parameter uncertainty problem related to the quantization density. The second contribution is to separate the term refer to the unknown transition probability in the sufficient condition for the distributed robust filter to satisfy the optimal disturbance attenuation level, and the corresponding distributed filter design method as well as explicit expression are given. The third contribution is the development of a distributed robust filtering algorithm based on a WSN to achieve plant state trajectory tracking well.

The rest of this article is organized below. In Section 2, system mathematical models and basic definitions are described. In Section 3, performance analyses are achieved. In Section 4, the distributed filter design method is given. In Section 5, two simulation 
examples are presented to verify the effectiveness of the proposed approach. In Section 6, conclusions are presented.

Notation. In this article, $\|\cdot\|$ denotes the Euclidean norm. For a matrix $P, P>0$ denotes a positive definite symmetric matrix. I denotes the unit matrix with compatible dimension. $\mathbb{E}\{\cdot\}$ is the mathematical expectation. Finally, the symbol " $*$ " represents a symmetric item in a matrix.

\section{Problem Statement}

Consider a class of discrete-time linear time-invariant systems as follows:

$$
\left\{\begin{array}{l}
x(k+1)=A x(k)+B w(k), \\
z(k)=M x(k),
\end{array}\right.
$$

where $x(k) \in \mathbb{R}^{n_{x}}$ is the system state with initial state $x_{0}, z(k)$ is the system output to be estimated, and $w(k) \in \mathbb{R}^{n_{w}}$ is the system interference, belongs to $w(k) \in \mathbb{R}^{n_{w}}$.

A distributed filtering framework based on WSNs is presented in Figure 1. The sensor node $i$ in a WSN can be described as follows

$$
y_{i}(k)=C_{i} x(k)+D_{i} v_{i}(k),
$$

where $y_{i}(k) \in \mathbb{R}^{n_{y}}$ is instantaneous sampling, $v_{i}(k)$ is the measurement noise belonging to $l_{2}[0, \infty)$. It should be noted that model (2) is a common linearization model that can be obtained using the linearization technique described in the literature $[33,34]$. Such a linearized measurement model, although inevitably subject to modelling errors, simplifies the design of the distributed filter. In addition, $A, B, M, C_{i}$ and $D_{i}$ are known matrices with appropriate dimensions.

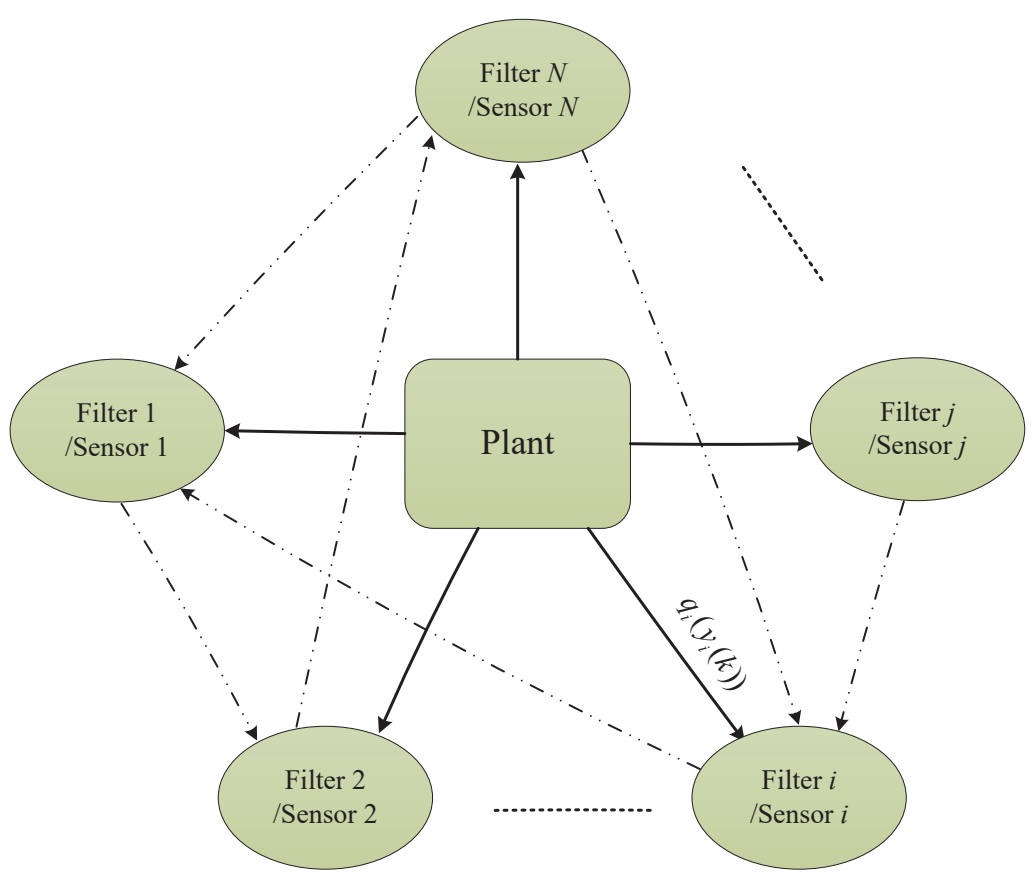

Figure 1. A distributed filtering framework based on WSNs. 
In this paper, a logarithmic quantizer $q_{i}(\cdot)$ is used to quantify the sensor measurement. $q_{i}\left(y_{i}(k)\right)$ represents the quantified measurement of sensor node $i$, and satisfies $q_{i}\left(-y_{i}(k)\right)=$ $-q_{i}\left(y_{i}(k)\right)$. Then, a logarithmic quantizer is explicitly defined as

$$
q_{i}\left(y_{i}(k)\right)= \begin{cases}y_{i}(k), & \text { if } \frac{1}{1+\delta_{i}} y_{i}(k)<y_{i}(k) \leq \frac{1}{1-\delta_{i}} y_{i}(k), y_{i}(k)>0 ; \\ 0, & \text { if } y_{i}(k)=0 \\ -q_{i}\left(-y_{i}(k)\right), & \text { if } y_{i}(k)<0\end{cases}
$$

where $\delta_{i}=\frac{1-\rho_{i}}{1+\rho_{i}}$ with quantization density $0<\rho_{i}<1$.

Inspired by the authors of [30], a logarithmic quantizer can be further expressed in the following form

$$
q_{i}\left(y_{i}(k)\right)=\left(I+\Delta_{q_{i}}(k)\right) y_{i}(k),
$$

where $\left|\Delta_{q_{i}}(k)\right| \leq \delta_{i}, i=1,2, \ldots, N$.

Assuming that $N$ filters are distributed in the target plant area, where the filters exchange information via a wireless network. Consider a network topology represented by a directed graph $\mathcal{G}^{r(k)}=\left(\mathcal{V}, \mathcal{E}, \mathcal{A}^{r(k)}\right)$ with the set of nodes $\mathcal{V}=\{1,2, \ldots, N\}$, edges $\mathcal{E} \subseteq \mathcal{V} \times \mathcal{V}$, and a weighted adjacency matrix $\mathcal{A}^{r(k)}=\left[a_{i j}\right]_{N \times N}^{r(k)}$. Nonnegative elements in the weighted adjacency matrix $a_{i j}$ satisfying the property $a_{i j}^{r(k)}>0 \Leftrightarrow(i, j) \in \mathcal{E}$, which means there is data exchange between node $j$ and node $i$. For all $i \in \mathcal{V}, a_{i i}^{r(k)}=0$. In addition, define $\mathcal{N}_{i}^{r(k)}=\{j \in \mathcal{V}:(i, j) \in \mathcal{E}\}$ to represent the set of neighboring nodes of node $i$, and matrix $\mathcal{L}^{r(k)}=\left(l_{i j}^{r(k)}\right)_{N \times N}$ to be the Laplacian matrix, where $l_{i i}^{r(k)}=\sum_{j \in \mathcal{N}_{i}^{r(k)}} a_{i j}^{r(k)}, l_{i j}^{r(k)}=$ $-a_{i j}^{r(k)}(i \neq j)$.

The $\{r(k)\}(0 \leq k<\infty)$ stands for Markov chain, and takes value in a finite set $\mathcal{S}=\left\{1,2, \ldots, n_{0}\right\}$. The transition probability matrix is $\Pi=\left[\pi_{s t}\right]_{n_{0} \times n_{0}}$ with

$$
\pi_{s t}=\operatorname{Prob}(r(k+1)=t \mid r(k)=s),
$$

where $\pi_{s t} \geq 0$, for all $s, t \in \mathcal{S}$, satisfy $\sum_{t=1}^{n_{0}} \pi_{s t}=1$.

Noting that not all elements of the transition probability matrix are completely known, a possible transition probability matrix is described as follows

$$
\Pi=\left[\begin{array}{ccccc}
\pi_{11} & ? & \pi_{13} & \cdots & ? \\
? & ? & ? & \cdots & \pi_{2 n_{0}} \\
\vdots & \vdots & \vdots & \ddots & \vdots \\
? & \pi_{n_{0} 2} & \pi_{n_{0} 3} & \cdots & \pi_{n_{0} n_{0}}
\end{array}\right]
$$

where "?" represents the unknown transition probability element in the transition probability matrix.

To simplify the analysis, we define the set $U^{s}=U_{m}^{s} \cup U_{u m}^{s}, s \in S$, where

$$
U_{m}^{s} \triangleq\left\{t: \pi_{s t} \text { is known }\right\}, U_{u m}^{s} \triangleq\left\{t: \pi_{s t} \text { is unknown }\right\} .
$$

If set $U_{m}^{s} \neq \varnothing$, elements in set $U_{m}^{s}$ can be relabeled as

$$
U_{m}^{s}=\left\{m_{1}^{s}, m_{2}^{s}, \ldots, m_{l}^{s}\right\}, 1 \leq l \leq S,
$$

where $m_{j}^{s}, j \in\{1,2, \ldots, l\}$ represents the $j$-th probability element in the $s$-th row in the transition probability matrix $\Pi$.

In a WSN, each node independently observes the same dynamic target in each sampling period. Taking into account the bandwidth limitation and digital signal transmission requirements in the network, the sensor observation needs to be quantized and encoded 
into a digital signal, and then sent to the corresponding filter node via the wireless network. At the network terminal, the filter node realizes real-time estimation for the target state based on the quantized observations and neighbor node' information. Construct the following distributed filter model with switching interconnection topology, where Markov chain describes the time-varying topologies,

$\left\{\begin{array}{l}\hat{x}_{i}(k+1)=A \hat{x}_{i}(k)+L_{i}^{r(k)}\left(q_{i}\left(y_{i}(k)\right)-C_{i} \hat{x}_{i}(k)\right)+K_{i}^{r(k)} \sum_{j \in \mathcal{N}_{i}^{r(k)}} a_{i j}^{r(k)}\left(\hat{x}_{j}(k)-\hat{x}_{i}(k)\right), \\ \hat{z}_{i}(k)=M \hat{x}_{i}(k),\end{array}\right.$

where $\hat{x}_{i}(k) \in \mathbb{R}^{n_{x}}$ and $\hat{z}_{i}(k) \in \mathbb{R}^{n_{z}}$ are the estimated state $x(k)$ and the estimated output $z(k)$, respectively. $L_{i}^{r(k)}$ and $K_{i}^{r(k)}$ are the filter gains to be determined.

To simplify the analysis, we replace $r(k)$ with $s$ and also replace $r(k+1)$ with $t$. Let $e_{i}(k)=x(k)-\hat{x}_{i}(k)$ and $\tilde{z}_{i}(k)=z(k)-\hat{z}_{i}(k)$, we can get the node filtering error dynamics

$$
\left\{\begin{aligned}
e_{i}(k+1)= & \left(A-L_{i}^{s} C_{i}\right) e_{i}(k)+B w(k)-L_{i}^{s} \Delta_{q_{i}}(k) C_{i} x(k) \\
& -\left(L_{i}^{s} D_{i}+L_{i}^{s} \Delta_{q_{i}}(k) D_{i}\right) v_{i}(k)-K_{i}^{s} \sum_{i \in \mathcal{N}_{i}^{s}} a_{i j}^{s}\left(e_{i}(k)-e_{j}(k)\right), \\
\tilde{z}_{i}(k)=M e_{i}(k) . &
\end{aligned}\right.
$$

Consider a WSN and filtering network with $N$ nodes, and define

$$
\begin{aligned}
& e(k)=\left[e_{1}^{T}(k), e_{2}^{T}(k), \ldots, e_{N}^{T}(k)\right]^{T}, \bar{x}(k)=\left[x^{T}(k), x^{T}(k), \ldots, x^{T}(k)\right]^{T}, \\
& \tilde{z}(k)=\left[\tilde{z}_{1}^{T}(k), \tilde{z}_{2}^{T}(k), \ldots, \tilde{z}_{N}^{T}(k)\right]^{T}, v(k)=\left[v_{1}^{T}(k), v_{2}^{T}(k), \ldots, v_{N}^{T}(k)\right]^{T}, \\
& \bar{A}=\operatorname{diag}\{A, A, \ldots, A\}, \bar{B}=\left[B^{T}, B^{T}, \ldots, B^{T}\right]^{T}, \\
& \bar{C}=\operatorname{diag}\left\{C_{1}, C_{2}, \ldots, C_{N}\right\}, \Delta_{q}(k)=\operatorname{diag}\left\{\Delta_{q_{1}}, \Delta_{q_{2}}, \ldots, \Delta_{q_{N}}\right\}, \\
& \bar{M}=\operatorname{diag}\{M, M, \ldots, M\}, \bar{D}=\left[D_{1}^{T}, D_{2}^{T}, \ldots, D_{N}^{T}\right]^{T}, \\
& \bar{L}^{s}=\operatorname{diag}\left\{L_{1}^{s}, L_{2}^{s}, \ldots, L_{N}^{s}\right\}, \bar{K}^{s}=\operatorname{diag}\left\{K_{1}^{s}, K_{2}^{s}, \ldots, K_{N}^{s}\right\} .
\end{aligned}
$$

Then, the following compact form can be obtained from Equation (10)

$$
\left\{\begin{array}{c}
e(k+1)=\left[\bar{A}-\bar{K}^{s} \mathcal{L}^{s}\right] e(k)-\left[\bar{L}^{s} \bar{C}+\Delta_{q}(k) \bar{C}\right] \bar{x}(k) \\
\quad-\left(\bar{L}^{s} \bar{D}+\Delta_{q}(k) \bar{D}\right) v(k)+\bar{B} w(k) \\
\tilde{z}(k)=\bar{M} e(k)
\end{array}\right.
$$

Define $\varsigma(k)=\left[\begin{array}{ll}\bar{x}^{T}(k) & e^{T}(k)\end{array}\right]^{T}$ and $\bar{w}(k)=\left[\begin{array}{cc}w^{T}(k) & v^{T}(k)\end{array}\right]^{T}$. According to Equations (1) and (12), the following augmented filtering error system (AFES) can be obtained

$$
\left\{\begin{array}{l}
\varsigma(k+1)=\mathscr{A}^{s} \varsigma(k)+\mathscr{B}^{s} \bar{w}(k), \\
\tilde{z}(k)=\mathcal{M}_{\varsigma}(k)
\end{array}\right.
$$

where

$$
\begin{aligned}
\mathscr{A}^{s} & =\left[\begin{array}{cc}
\bar{A} & 0 \\
-\bar{L}^{s} \Delta_{q}(k) \bar{C} & \bar{A}-\bar{L}^{s} \bar{C}-\bar{K}^{s} \mathcal{L}^{s}
\end{array}\right], \\
\mathscr{B}^{s} & =\left[\begin{array}{cc}
\bar{B} & 0 \\
\bar{B} & -\bar{L}^{s} \bar{D}-\bar{L}^{s} \Delta_{q}(k) \bar{D}
\end{array}\right], \mathscr{M}=\left[\begin{array}{ll}
0 & \bar{M}
\end{array}\right] .
\end{aligned}
$$

This study aims to construct a distributed state estimator (9) over WSNs, such that AFES (13) satisfies the following conditions: 
(a) For all $\varsigma(0)$, the AFES (13) with $\bar{w}(k)=0$ is exponentially mean-square stable if there exist scalars $\lambda \geq 1$ and $0<\tau<1$, such that

$$
\lim _{k \rightarrow \infty} \mathbb{E}\left\|_{\varsigma}(k)\right\| \leq \lambda \tau^{k} \mathbb{E}\{\|\varsigma(0)\|\}
$$

(b) Given a disturbance attenuation level $\gamma>0$, the AFES (13) with $\bar{w}(k) \neq 0$ exhibits $H_{\infty}$ robust performance, if there are definite symmetric matrices $R^{s}>0, s \in \mathcal{S}$, and satisfies the following constraint:

$$
J:=\|\tilde{z}(k)\|_{[0, N-1]}^{2}-\gamma^{2}\|\bar{w}(k)\|_{[0, N-1]}^{2}-\gamma^{2} \varsigma^{T}(0) R^{s} \varsigma(0)<0 .
$$

Lemma 1. (see the work of De et al. [35]) If the matrices $A, Q=Q^{T}$ and $P>0$ meet the following inequality constraint,

$$
A^{T} P A-Q<0,
$$

Then, for any matrix $G$ with appropriate dimension, the following inequality holds,

$$
\left[\begin{array}{cc}
-Q & * \\
G^{T} A & P-G-G^{T}
\end{array}\right]<0 .
$$

Lemma 2. (see the work of Yang et al. [36]) If there are matrices $Q, R$ and $F(t)$ with appropriate dimensions, satisfying $F^{T}(t) F(t) \leq I$, and

$$
Q F(t) R+R^{T} F^{T}(t) Q^{T}<0 .
$$

Then, a constant $\varepsilon>0$ can be found such that

$$
\varepsilon Q Q T+\varepsilon^{-1} R^{T} R<0 .
$$

\section{Stability and Robustness Analysis}

In this section, we give sufficient conditions to guarantee that the AFES (13) is mean square exponentially stable and robust.

Theorem 1. Given disturbance attenuation level $\gamma>0$. Suppose there exists a family of positive definite matrix $P^{s}>0$, which satisfies the initial condition $\varsigma^{T}(0) P^{s} \varsigma(0) \leq \gamma^{2} \varsigma^{T}(0) R^{s} \varsigma(0), s=$ $1,2, \ldots, n_{0}$ and the following inequality constraint

$$
\left[\begin{array}{cccc}
-P^{s} & * & * & * \\
0 & -\gamma^{2} I & * & * \\
\bar{P}^{s} \mathscr{A}^{s} & \bar{P}^{s} \mathscr{B}^{s} & -\bar{P}^{s} & * \\
\mathscr{M} & 0 & 0 & -I
\end{array}\right]<0
$$

where $\bar{P}^{s}=\sum_{t=1}^{n_{0}} \pi_{s t} P^{t}$. Then, the AFES (13) achieves the desired exponential stability and $H_{\infty}$ roubst performance.

Proof. The proof is given in Appendix A.

\section{Distributed Filter Design}

In this section, we give explicit expressions for the distributed filter gains.

Theorem 2. The AFES (13) with discrete linear system (1) and distributed filter (9) achieves the desired disturbance attenuation level $\gamma>0$, if there are positive definite matrices $P_{1}^{s}>0, P_{2}^{s}>$ 0 , diagonal matrices $X^{s}, Y^{s}, V_{2}^{s}$, matrices $W_{i}^{s}, V_{1}^{s}, i=1,2,3,4,5,6,7,8, s=1,2, \ldots, n_{0}$, and a 
constant $\varepsilon>0$, satisfying $\varsigma^{T}(0) P^{s} \varsigma(0) \leq \gamma^{2} \varsigma^{T}(0) R^{s} \varsigma(0), s=1,2, \ldots n_{0}$ and the following linear matrix inequality constraints

$$
\begin{gathered}
{\left[\begin{array}{cccccccc}
\Xi_{11}^{s} & * & * & * & * & * & * & * \\
0 & \Xi_{22}^{s} & * & * & * & * & * & * \\
0 & 0 & \Xi_{33}^{s} & * & * & * & * & * \\
\Xi_{41}^{s} & 0 & 0 & \Xi_{44}^{s} & * & * & * & * \\
\Xi_{51}^{s} & 0 & \Xi_{53}^{s} & 0 & \Xi_{55}^{s} & * & * & * \\
0 & \Xi_{62}^{s} & \Xi_{63}^{s} & \Xi_{64}^{s} & 0 & \Xi_{66}^{s} & * & * \\
0 & \Xi_{72}^{s} & 0 & 0 & 0 & 0 & \Xi_{77}^{s} & * \\
0 & 0 & 0 & 0 & 0 & \Xi_{86}^{s} & 0 & \Xi_{88}^{s}
\end{array}\right]<0,} \\
\operatorname{diag}\left\{-W_{1}^{s},-W_{2}^{s},-W_{3}^{s},-W_{4}^{s}, P_{1}^{t}-W_{5}^{s}, P_{2}^{t}-W_{6}^{s}-W_{7}^{s},-W_{8}^{s}\right\}<0, t \in U_{u m}^{s},
\end{gathered}
$$

where

$$
\begin{aligned}
& \beta^{s}=\left(1-\sum_{t \in U_{m}^{s}}^{n_{0}} \pi_{s t}\right), \Xi_{11}^{s}=-P_{1}^{s}+\beta^{s} W_{1}^{s}+\varepsilon^{-1} \bar{C}^{T} \bar{C}, \Xi_{22}^{s}=-P_{2}^{s}+\beta^{s} W_{2}^{s}, \\
& \Xi_{33}^{s}=-\gamma^{2} I+\beta^{s} W_{3}^{s}, \Xi_{41}^{s}=\varepsilon^{-1} \bar{D}^{T} \bar{C}, \Xi_{44}^{s}=-\gamma^{2} I+\beta^{s} W_{4}^{s}+\varepsilon^{-1} \bar{D}^{T} \bar{D}, \\
& \Xi_{51}^{s}=V_{1}^{s} \bar{A}, \Xi_{53}^{s}=V_{1}^{s} \bar{B}, \Xi_{64}^{s}=-Y^{s} \bar{D}, \Xi_{55}^{s}=\sum_{t \in U_{m}^{s}}^{n_{0}} \pi_{s t} P_{1}^{t}-V_{1}^{s}-V_{1}^{s T}+\beta^{s} W_{5}^{s}, \\
& \Xi_{62}^{s}=V_{2}^{s} \bar{A}-Y^{s} \bar{C}-X^{s} \mathcal{L}^{s}, \Xi_{63}^{s}=V_{2}^{s} \bar{B}, \Xi_{66}^{s}=\sum_{t \in U_{m}^{s}}^{n_{0}} \pi_{s t} P_{2}^{t}-V_{2}^{s}-V_{2}^{s T}+\beta^{s} W_{6}^{s}, \\
& \Xi_{72}^{s}=\bar{M}, \Xi_{77}^{s}=-I+\beta^{s} W_{7}^{s}, \Xi_{86}^{s}=-\varepsilon H^{T} Y^{s T}, \Xi_{88}^{s}=-\varepsilon I+\beta^{s} W_{8}^{s} .
\end{aligned}
$$

Then, the distributed $H_{\infty}$ filtering problem can be solved, where the distributed filter parameters are explicitly described as

$$
\bar{L}^{s}=\left(V_{2}^{s}\right)^{-1} Y^{s}, \bar{K}^{s}=\left(V_{2}^{s}\right)^{-1} X^{s} .
$$

Proof. The proof is presented in Appendix B.

\section{Numerical Example}

In this section, two simulation examples are described to demonstrate the validity of the results presented in this paper.

Example 1. For plant (1), we select the following parameters in this example,

$$
A=\left[\begin{array}{cc}
0.4 & 0.8 \\
0.6 & -0.5
\end{array}\right], B=\left[\begin{array}{l}
0.5 \\
0.4
\end{array}\right], M=\left[\begin{array}{ll}
2 & -1
\end{array}\right] .
$$

Assume that the distributed filtering network includes three switchable communication topologies, with the associated adjacency matrices

$$
\mathcal{A}_{1}=\left[\begin{array}{llll}
0 & 1 & 1 & 1 \\
0 & 0 & 1 & 0 \\
1 & 0 & 0 & 0 \\
0 & 1 & 0 & 0
\end{array}\right], \mathcal{A}_{2}=\left[\begin{array}{llll}
0 & 0 & 1 & 0 \\
0 & 0 & 0 & 1 \\
1 & 1 & 0 & 1 \\
0 & 0 & 1 & 0
\end{array}\right], \mathcal{A}_{3}=\left[\begin{array}{llll}
0 & 1 & 0 & 1 \\
1 & 0 & 1 & 0 \\
0 & 1 & 0 & 1 \\
1 & 0 & 0 & 0
\end{array}\right],
$$

and the corresponding parameters are selected as follows

$$
\begin{aligned}
& C_{1}=\left[\begin{array}{ll}
0.1 & 0.8
\end{array}\right], C_{2}=\left[\begin{array}{ll}
0.1 & 0.2
\end{array}\right], C_{3}=\left[\begin{array}{ll}
0.2 & 0.4
\end{array}\right], \\
& C_{4}=\left[\begin{array}{ll}
0.5 & 0.4
\end{array}\right], D_{1}=0.2, D_{2}=0.1, D_{3}=0.2, D_{4}=0.1 .
\end{aligned}
$$


For the logarithmic quantizer (3), the quantizer densities are chosen as $\rho_{1,3}=0.6667$, $\rho_{2,4}=0.4288$, and system disturbance and measurement noise are $w(k)=e^{-0.2 k} \sin (k)$ and $v_{i}(k)=N\left(0, \frac{1}{k^{2}}\right), i=1,2,3,4$, respectively.

In this simulation example, four cases of state transition probability matrix are considered

$$
\begin{aligned}
& \text { Case } 1: \Pi=\left[\begin{array}{ccc}
0.4 & 0.2 & 0.4 \\
0.2 & 0.3 & 0.5 \\
0.7 & 0.1 & 0.2
\end{array}\right] \text {, Case } 2: \Pi=\left[\begin{array}{ccc}
0.4 & ? & ? \\
0.2 & 0.3 & 0.5 \\
0.7 & 0.1 & 0.2
\end{array}\right] \text {, } \\
& \text { Case } 3: \Pi=\left[\begin{array}{ccc}
0.4 & 0.2 & 0.4 \\
? & 0.3 & ? \\
? & 0.1 & ?
\end{array}\right] \text {, Case } 4: \Pi=\left[\begin{array}{ccc}
? & ? & 0.4 \\
0.2 & ? & ? \\
? & 0.1 & ?
\end{array}\right] \text {. }
\end{aligned}
$$

Moreover, let $r(0)=1$, and the system state and distributed filter state are both zero.

We use Matlab 2019b Yalmip toolbox to solve the $H_{\infty}$ filtering problem (22) and (23) with a given robust performance index $\gamma=7.9$. Figures $2-5$ show the corresponding simulation results. Figure 2 shows a possible evolution of Markov chain. Figure 3 presents a comparison of quantified and non-quantified measurements in case 2 and Figure 4 shows the output estimation from the distributed filtering network. Figure 5 shows the evolution of root-mean-square error of the filter in four different state transition probability matrices, which implies that the developed distributed filter can be applied to two cases, that is, the state transition probability matrix is totally known and partially unknown.

In addition, we explored the relationship between quantization density, the unknown degree of the topolgy switching matrix, and $H_{\infty}$ robustness performance. Table 1 provides the comparison results of the robust performance index $\gamma$ and the solving time in four different topologies switching probability matrices. Table 2 presents the relationship between different quantization density and robust performance. It can be seen from Tables 1 and 2 that the more complete the state transition probability information and the higher the quantization density, the smaller the interference attenuation level $\gamma$ obtained; that is, the better robust performance of the filtering network achieved.

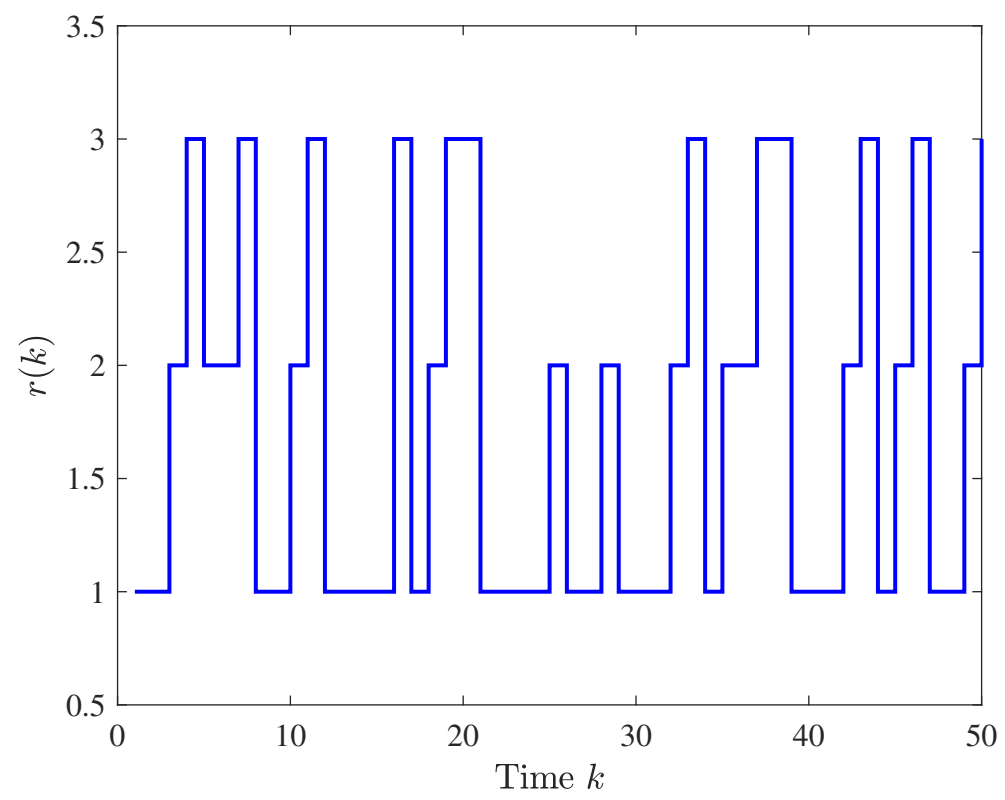

Figure 2. Evolution of $r(k)$ in Case 2. 

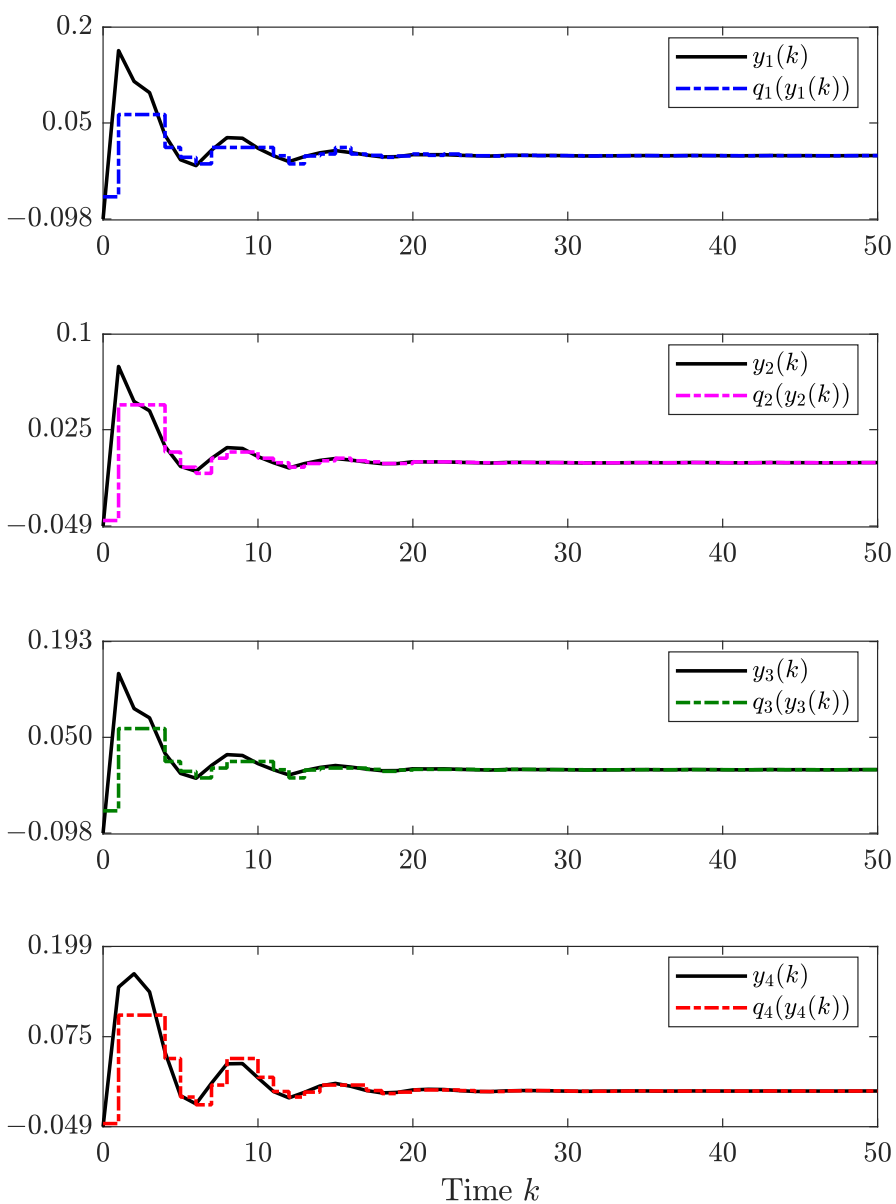

Figure 3. Measurement and quantized measurement of sensor $i$ in Case 2, $i=1,2,3,4$.

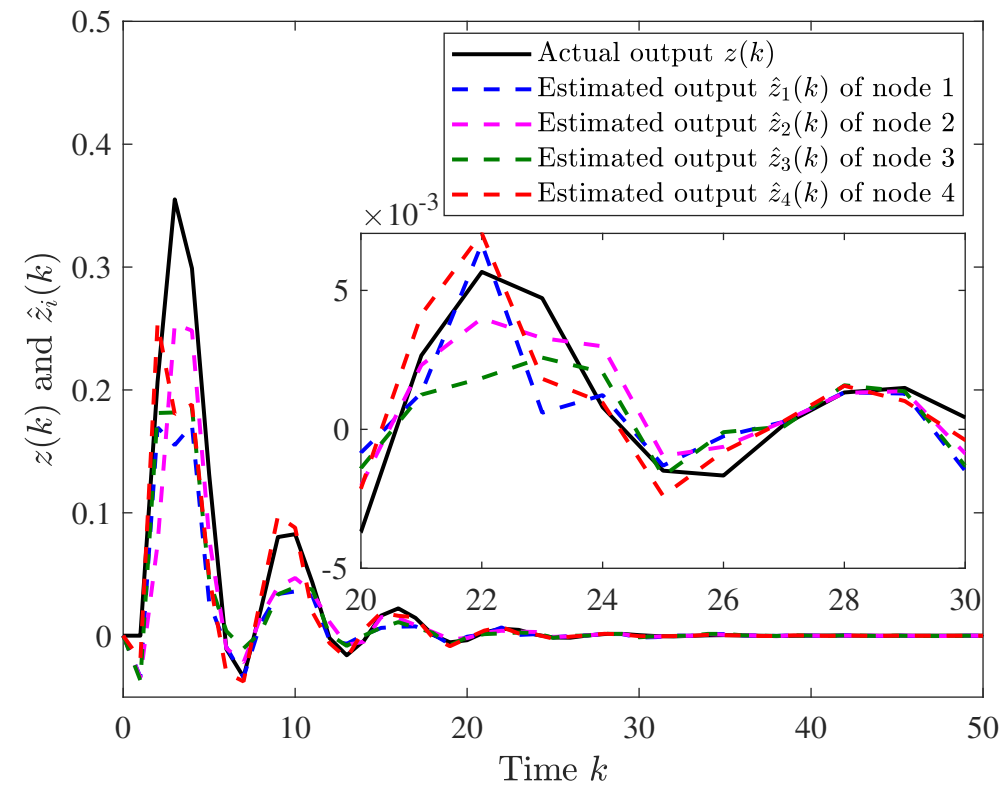

Figure 4. Evolution of plant output $z(k)$ and estimated output $\hat{z}_{i}(k)$ in Case $2, i=1,2,3,4$. 


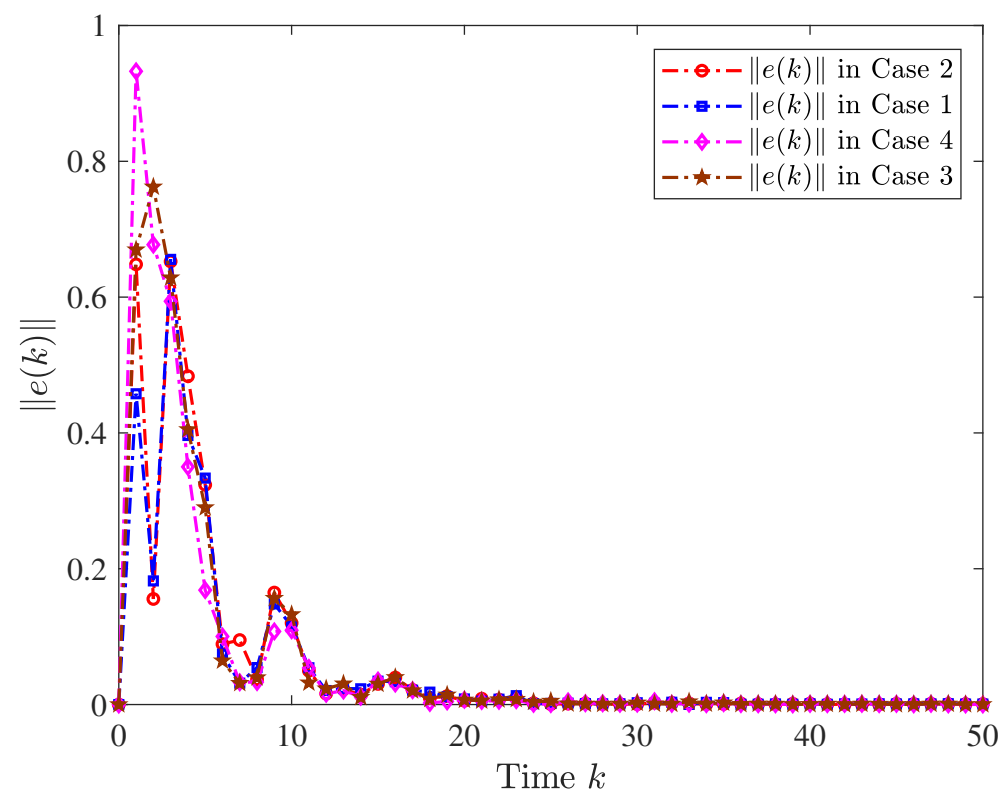

Figure 5. Evolution of filtering error $\|e(k)\|$ for four cases.

Table 1. The $H_{\infty}$ performance index $\gamma$ and solver time for different quantization density $\rho_{i}$ in case 2 .

\begin{tabular}{ccccc}
\hline Case $\boldsymbol{i}$ & Case 1 & Case 2 & Case 3 & Case 4 \\
\hline Minimize $\gamma$ & 7.9716 & 7.9744 & 9.3631 & 10.3198 \\
Solver time $(\mathrm{s})$ & 0.3851 & 0.4820 & 0.6714 & 0.7005 \\
\hline
\end{tabular}

Table 2. The $H_{\infty}$ performance index $\gamma$ and solver time for different quantization density $\rho_{i}$ in case 2 .

\begin{tabular}{cccccc}
\hline $\boldsymbol{\rho}_{\mathbf{1}}$ & $\mathbf{0 . 8}$ & $\mathbf{0 . 6}$ & $\mathbf{0 . 5}$ & $\mathbf{0 . 4}$ & $\mathbf{0 . 1}$ \\
\hline$\rho_{2}$ & 0.9 & 0.7 & 0.6 & 0.2 & 0.1 \\
$\rho_{3}$ & 0.7 & 0.5 & 0.3 & 0.2 & 0.1 \\
$\rho_{4}$ & 0.8 & 0.7 & 0.4 & 0.1 & 0.1 \\
Minimize $\gamma$ & 7.8325 & 7.8505 & 7.8847 & 8.0482 & 8.3237 \\
\hline
\end{tabular}

Example 2. In this example, the developed distributed filtering approach is applied to address the state estimation problem for a continuously stirred tank reactor. Considering this reactor, a motor-driven stirrer can stir the reactor feed $R_{\text {in }}$ and then obtain the reactor product $R_{\text {out }}$ at the reactor bottom. The concentration of the reactor product plays a key role in obtaining the desired product and should be monitored at all times. Although concentration monitoring can be achieved using traditional chemical measurement analysis methods, using such direct measurements can increase the cost. One solution is to estimate the reactor product concentration by using the proposed distributed $H_{\infty}$ state estimation method, which is achieved by collecting the reactor temperature $R_{T}$ through the four sensors placed at different locations, as shown in Figure 6. 


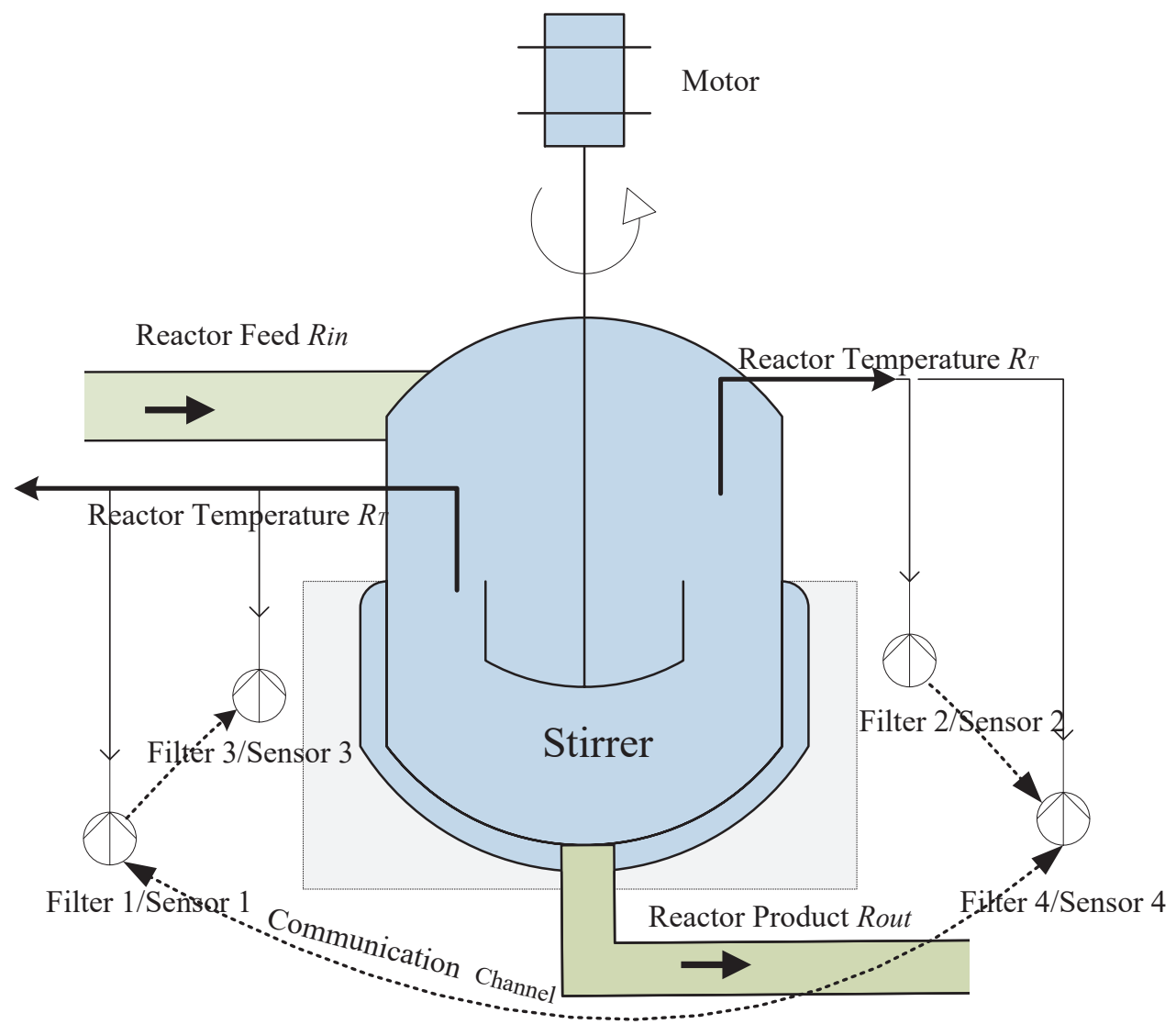

Figure 6. A continuous stirred tank reactor with four sensor nodes.

Let us assume that, at instant $k, x_{1}(k)$ and $x_{2}(k)$ represent the concentrations of the transport from $R_{i n}$ and the product $R_{\text {out }}$, respectively, and $x_{3}(k)$ represents the reactor temperature $x_{3}(k)$. Four sensors were used to monitor the reactor temperature in order to increase the estimation system's robustness against sensor failures and to improve estimation performance. Then, the discrete version of the linearized state space model of the reactor with a sampling period of $T=0.5 \mathrm{~s}$ can be obtained as follows:

$$
\begin{aligned}
A & =\left[\begin{array}{ccc}
0.622 & 0.008 & 0.014 \\
0.195 & 0.623 & -0.001 \\
-0.175 & 0.771 & 0.636
\end{array}\right], B=\left[\begin{array}{l}
0.001 \\
0.398 \\
0.226
\end{array}\right], \\
M & =\left[\begin{array}{lll}
0 & 1 & 0
\end{array}\right], C_{i}=\left[\begin{array}{lll}
0 & 0 & 1
\end{array}\right], D_{i}=0.1, i=1,2,3,4 .
\end{aligned}
$$

In addition, assume that $r(0)=1$, the disturbance input $w(k)=N(0,0.5)$ and measurement noise $v_{i}(k)=1 / k^{3}, i=1,2,3,4$ and the other parameters are the same as in Example 1.

By solving the $H_{\infty}$ filtering problem (22) and (23) with a given robust performance index $\gamma=15.2$, and the corresponding simulation results are shown in Figures 7-9. Figure 7 shows a possible Markov chain evolution that represents a random switching sequence of communication topologies in Case 3. Figure 8 presents a comparison of quantified and non-quantified measurements at sensor nodes. Figure 9 shows the actual output of the system and the estimation of filter nodes, which demonstrates the effectiveness and potential applicability of the developed distributed robust filtering approach. 


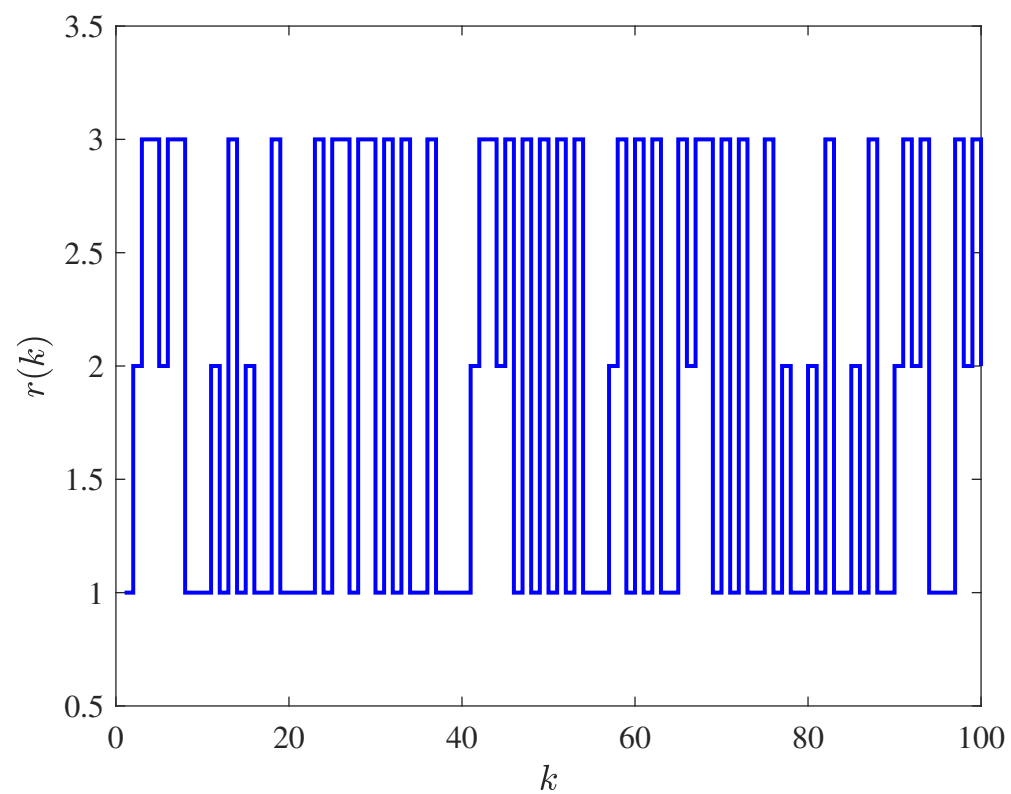

Figure 7. Evolution of $r(k)$ in Case 3.
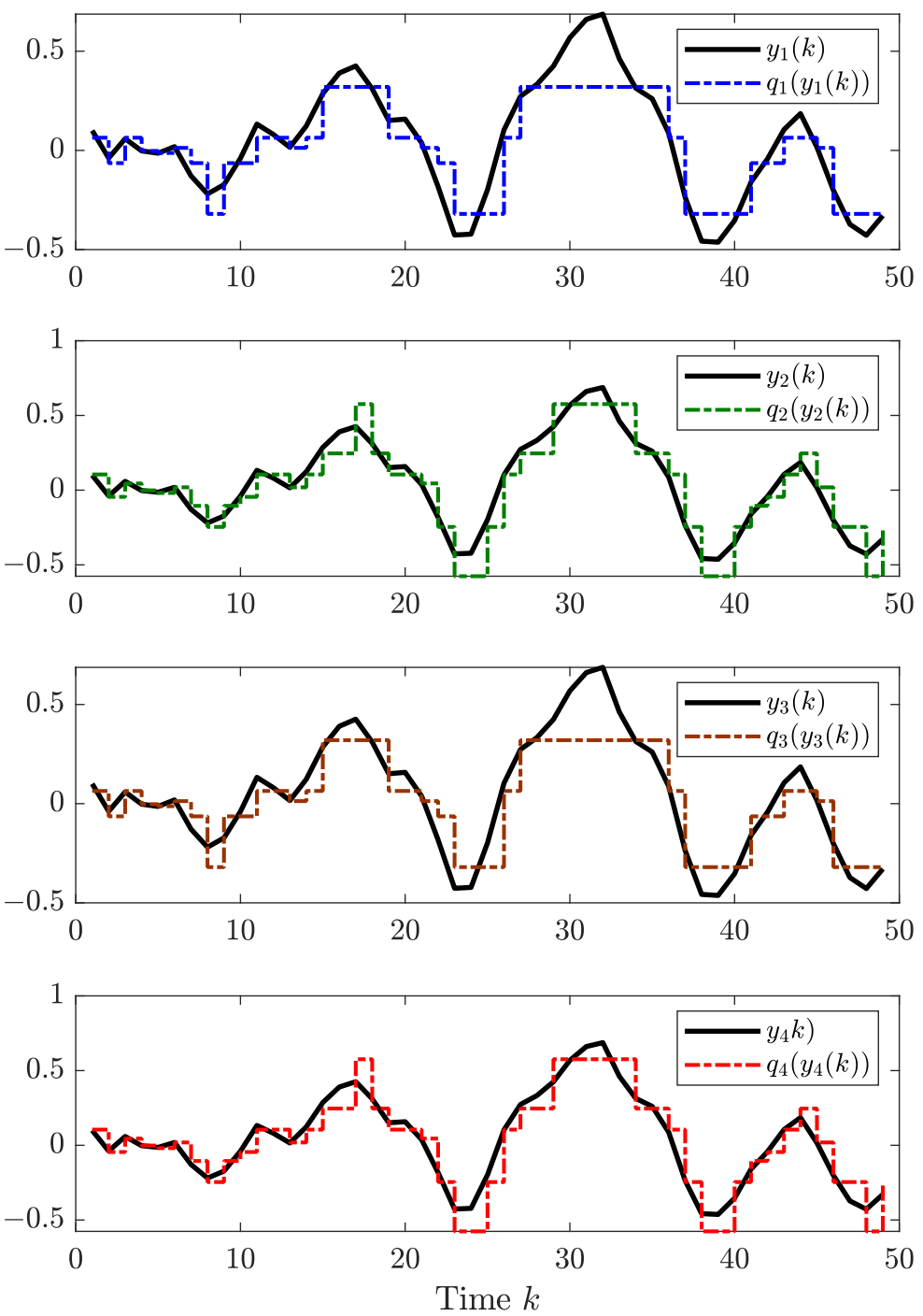

Figure 8. The actual measurement $y(k)$, and the quantified measurement $q_{i}\left(y_{i}(k)\right), i=1,2,3,4$. 


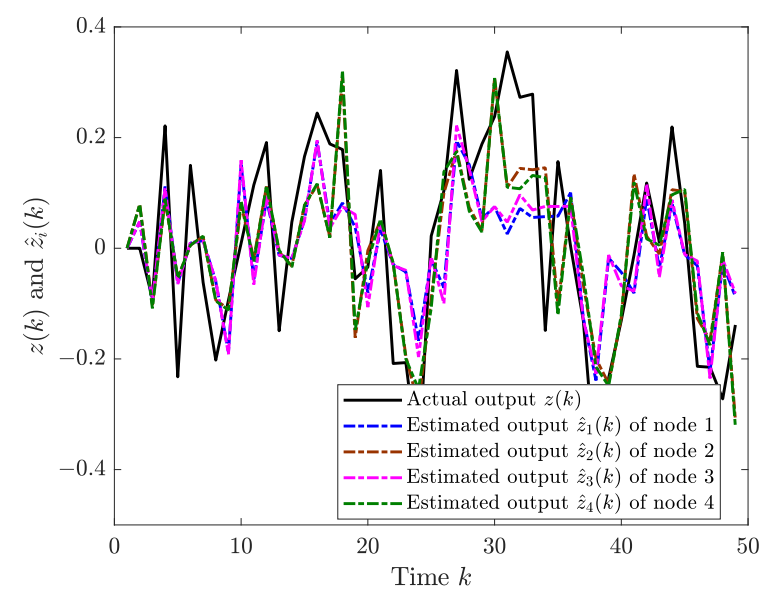

Figure 9. Evolution of system output $z(k)$ and estimated output $\hat{z}_{i}(k)$ in Case $3, i=1,2,3,4$.

With the two simulation examples above, we have demonstrated the effectiveness of the developed distributed $H_{\infty}$ robust filter. However, there are still some limitations to this distributed estimator design method. For example, we reselect the external disturbance as $w(k)=e^{0.01 k}$ and keep the other system parameters the same as in simulation Example 2 . By solving the $H_{\infty}$ filtering problem (22) and (23) with a given robust performance index $\gamma=18.1$, we obtained the following corresponding distributed estimator gains for the Case 4 .

$$
\begin{aligned}
& K_{11}^{1}=\left[\begin{array}{lll}
0.1480 & 0.0021 & -0.0043 \\
0.2352 & 0.0480 & -0.0670 \\
0.4503 & 0.0622 & -0.1066
\end{array}\right], K_{22}^{1}=\left[\begin{array}{lll}
0.3338 & 0.0045 & -0.0095 \\
0.5146 & 0.0966 & -0.1352 \\
0.9766 & 0.1245 & -0.2142
\end{array}\right] \text {, } \\
& K_{33}^{1}=\left[\begin{array}{lll}
0.4239 & 0.0061 & -0.0127 \\
0.6874 & 0.1437 & -0.2004 \\
1.3438 & 0.1866 & -0.3196
\end{array}\right], K_{44}^{1}=\left[\begin{array}{lll}
0.1739 & 0.0022 & -0.0047 \\
0.2961 & 0.0489 & -0.0687 \\
0.5557 & 0.0624 & -0.1082
\end{array}\right] \text {, } \\
& K_{11}^{2}=\left[\begin{array}{lll}
0.2027 & 0.0042 & -0.0069 \\
0.5876 & 0.0380 & -0.0668 \\
0.5113 & 0.0741 & -0.1234
\end{array}\right], K_{22}^{2}=\left[\begin{array}{lll}
0.2000 & 0.0045 & -0.0072 \\
0.5462 & 0.0374 & -0.0657 \\
0.4090 & 0.0735 & -0.1214
\end{array}\right] \text {, } \\
& K_{33}^{2}=\left[\begin{array}{lll}
0.1817 & 0.0041 & -0.0066 \\
0.5663 & 0.0377 & -0.0662 \\
0.4741 & 0.0739 & -0.1226
\end{array}\right], K_{44}^{2}=\left[\begin{array}{lll}
0.3935 & 0.0086 & -0.0137 \\
1.1727 & 0.0760 & -0.1335 \\
0.9378 & 0.1467 & -0.2441
\end{array}\right] \text {, } \\
& K_{11}^{3}=\left[\begin{array}{lll}
0.2527 & 0.0058 & -0.0092 \\
0.7530 & 0.0497 & -0.0882 \\
0.6067 & 0.1069 & -0.1730
\end{array}\right], K_{22}^{3}=\left[\begin{array}{lll}
0.1899 & 0.0043 & -0.0068 \\
0.4702 & 0.0327 & -0.0580 \\
0.3496 & 0.0714 & -0.1148
\end{array}\right] \text {, } \\
& K_{33}^{3}=\left[\begin{array}{lll}
0.1009 & 0.0022 & -0.0036 \\
0.2046 & 0.0159 & -0.0282 \\
0.1382 & 0.0358 & -0.0571
\end{array}\right], K_{44}^{3}=\left[\begin{array}{lll}
0.3564 & 0.0080 & -0.0127 \\
1.0153 & 0.0664 & -0.1179 \\
0.8152 & 0.1424 & -0.2307
\end{array}\right] \text {, } \\
& L_{11}^{1}=\left[\begin{array}{l}
0.0313 \\
0.6849 \\
1.4808
\end{array}\right], L_{22}^{1}=\left[\begin{array}{l}
0.0313 \\
0.6849 \\
1.4809
\end{array}\right], L_{33}^{1}=\left[\begin{array}{l}
0.0312 \\
0.6849 \\
1.4809
\end{array}\right], L_{44}^{1}=\left[\begin{array}{l}
0.0312 \\
0.6849 \\
1.4808
\end{array}\right] \text {, } \\
& L_{11}^{2}=\left[\begin{array}{l}
0.0313 \\
0.6849 \\
1.4808
\end{array}\right], L_{22}^{2}=\left[\begin{array}{l}
0.0312 \\
0.6849 \\
1.4809
\end{array}\right], L_{33}^{2}=\left[\begin{array}{l}
0.0312 \\
0.6849 \\
1.4809
\end{array}\right], L_{44}^{2}=\left[\begin{array}{l}
0.0313 \\
0.6849 \\
1.4808
\end{array}\right] \text {, } \\
& L_{11}^{3}=\left[\begin{array}{l}
0.0312 \\
0.6849 \\
1.4809
\end{array}\right], L_{22}^{3}=\left[\begin{array}{l}
0.0313 \\
0.6849 \\
1.4809
\end{array}\right], L_{33}^{3}=\left[\begin{array}{l}
0.0313 \\
0.6849 \\
1.4809
\end{array}\right], L_{44}^{3}=\left[\begin{array}{l}
0.0312 \\
0.6849 \\
1.4809
\end{array}\right] \text {. }
\end{aligned}
$$


Similarly, Figure 10 presents a comparison of quantified and non-quantified measurements at sensor nodes. Figure 11 shows the actual output of the system and the estimation of filter nodes. According to Figure 11, it can be seen that the distributed filters cannot track the state trajectory of the system well. This is owing to the fact that the state response trajectory of the plant is relatively "flat" and the quantization density of the quantizer used is too small. As a result, the quantized signal does not reflect the actual sensor measurement signal well, leading to undesirable estimation results. On the other hand, our distributed filter design method cannot deal with the fact that the elements of a certain row in the transition probability matrix are totally unknown. In other words, our method implicitly requires that there is at least one known probability element in each row of the transition probability matrix. Furthermore, the distributed robust filter developed in this paper can only be applied to the state estimation for linear systems, and our distributed filtering algorithm may be invalid when there are stochastic non-linearities, time lags, etc., in the system.
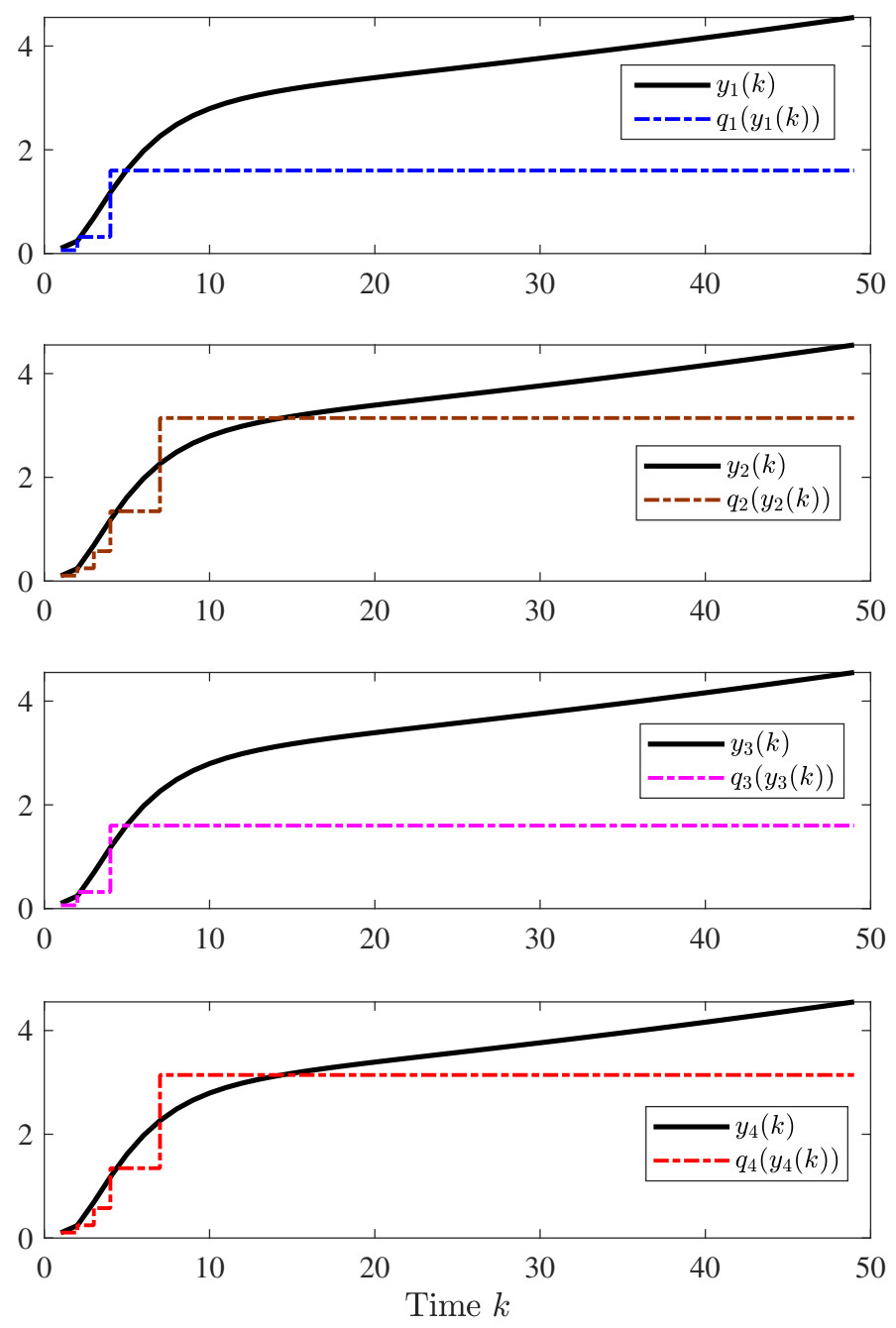

Figure 10. The actual measurement $y(k)$, and the quantified measurement $q_{i}\left(y_{i}(k)\right), i=1,2,3,4$. 


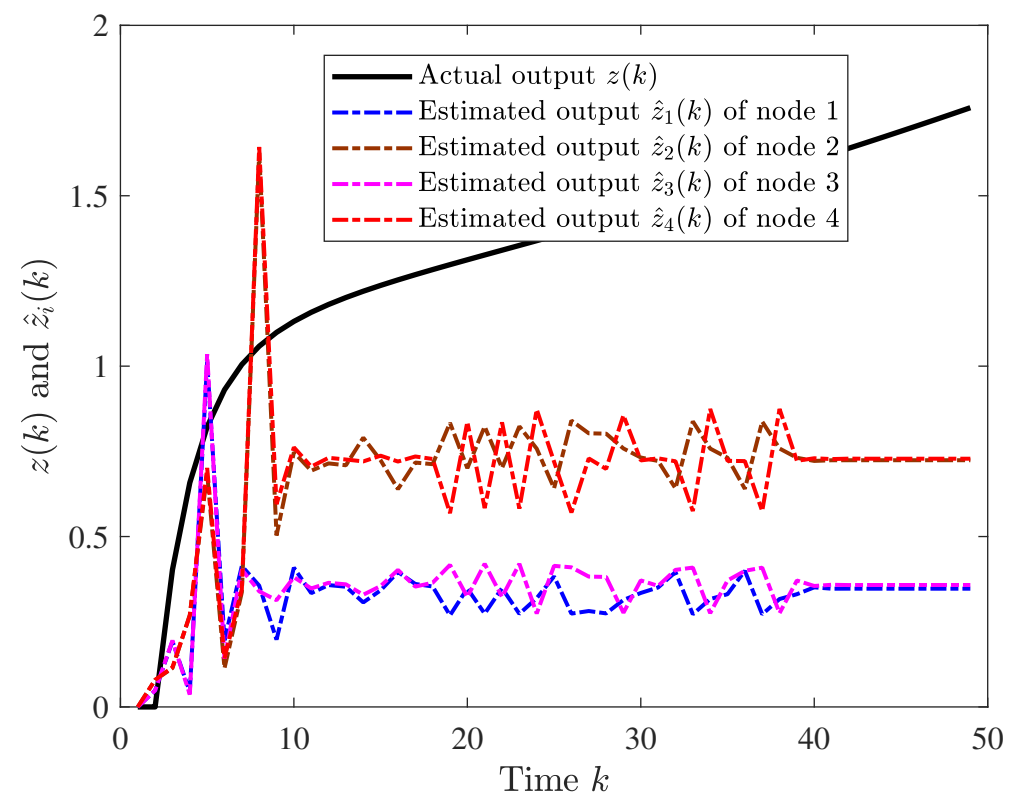

Figure 11. Evolution of system output $z(k)$ and estimated output $\hat{z}_{i}(k)$ in Case $4, i=1,2,3,4$.

\section{Conclusions}

This paper solved the distributed filtering problem with sensor quantization and stochastic switching topology over WSNs. A separate and static logarithmic quantizer for each measurement channel was modeled, and the sector bound approach was used to describe the quantization error. At the same time, by using the free-connection weighting matrix technology, the sufficient conditions for the existence of the distributed filter were split into two sets of linear matrix inequality constraints, one referring to known probability information, and the other referring to unknown probability information. The simulation example shows the performance analysis for the distributed filter network in different sensor quantization density and switching topologies, and verifies the effectiveness of the developed approach. At present, the application of WSN is becoming more and more promising, and the application of distributed filtering algorithm in smart grid may be one of our future research directions.

Author Contributions: Conceptualization, F.Z. and S.D.; funding acquisition, S.D.; investigation, F.Z.; methodology, F.Z. and S.D.; project administration, F.Z. and S.D.; software, F.Z.; supervision, S.D.; writing—original draft preparation, F.Z.; writing—review and editing, S.D. Both authors have read and agreed to the published version of the manuscript.

Funding: This research was funded by the National Natural Science Foundation of China under Grant 61873112.

Institutional Review Board Statement: Not applicable.

Informed Consent Statement: Not applicable.

Data Availability Statement: All data and programs were created by us. Please contact the corresponding author of this article for relevant information.

Acknowledgments: The authors thank the anonymous reviewers for their careful reading and constructive comments.

Conflicts of Interest: The authors declare no conflicts of interest. 


\section{Appendix A. Proof of Theorem 1}

Proof. First, let us consider $\bar{w}(k)=0$ and select the Lyapunov function as follows:

$$
V(k)=\varsigma^{T}(k) P(r(k)) \varsigma(k) .
$$

Then, we have

$$
\mathbb{E}\{\Delta V(k)\}=\varsigma^{T}(k) \mathscr{A}^{s T} \bar{P}^{s} \mathscr{A}^{s} \varsigma(k)-\varsigma^{T}(k) P^{s} \varsigma(k) .
$$

We have

$$
\mathbb{E}\{\Delta V(k)\} \leq \varsigma^{T}(k) \Lambda^{s} \varsigma(k),
$$

where

$$
\Lambda^{s}=\mathscr{A}^{s T} \bar{P}^{s} \mathscr{A}^{S}-P^{s} .
$$

Applying Schur complementary inducements to inequality (21) in Theorem 1 yields $\Lambda^{s}<0, s=1,2, \ldots, n_{0}$. For all $s \in \mathcal{S}$, let $\lambda_{0}=\max \left[\lambda_{\max }\left(\Lambda^{s}\right)\right]$; clearly, $\lambda_{0}<0$. Clearly, there is

$$
\mathbb{E}\{\Delta V(k)\} \leq \lambda_{0} \mathbb{E}\left\{\|\varsigma(k)\|^{2}\right\} .
$$

Let $\sigma>0, \lambda_{1}=\max \left[\lambda_{\max }\left(P^{s}\right)\right], s=1,2,3, \ldots n_{0}$. From inequality (A5), one has

$$
\begin{aligned}
\frac{1}{\sigma^{k+1}} \mathbb{E}\{V(k+1)\}-\frac{1}{\sigma^{k}} \mathbb{E}\{V(k)\}= & \frac{1}{\sigma^{k+1}}(\mathbb{E}\{V(k+1)\}-\mathbb{E}\{V(k)\}) \\
& +\frac{1}{\sigma^{k}}\left(\frac{1}{\sigma}-1\right) \mathbb{E}\{V(k)\} \\
\leq & \frac{\lambda_{0}}{\sigma^{k+1}} \mathbb{E}\left\{\|\varsigma(k)\|^{2}\right\}+\frac{1}{\sigma^{k}}\left(\frac{1}{\sigma}-1\right) \mathbb{E}\{V(k)\} \\
\leq & \frac{1}{\sigma^{k}}\left(\frac{\lambda_{0}}{\sigma^{k}}+\left(\frac{1}{\sigma}-1\right) \lambda_{1}\right) \mathbb{E}\left\{\|\varsigma(k)\|^{2}\right\} .
\end{aligned}
$$

Note that for $\sigma=1$,

$$
\frac{1}{\sigma^{k}}\left(\frac{\lambda_{0}}{\sigma^{k}}+\left(\frac{1}{\sigma}-1\right) \lambda_{1}\right)=\lambda_{0}<0
$$

Given the continuity, a constant $0<\sigma_{0}<1$ can be found. Further, it satisfies $\left(1 / \sigma^{k}\right)\left[\left(\lambda_{0} / \sigma^{k}\right)+\left(\lambda_{1} / \sigma\right)-\lambda_{1}\right]<0$.

Then, from inequality (A6), we have

$$
\frac{1}{\sigma_{0}^{k+1}} \mathbb{E}\{V(k+1)\}-\frac{1}{\sigma_{0}^{k}} \mathbb{E}\{V(k)\} \leq 0,
$$

which implies that

$$
\frac{1}{\sigma_{0}^{k}} \mathbb{E}\{V(k)\} \leq V(0)=\left.\varsigma^{T}(0) P^{s} \varsigma(0)\right|_{s=0} \leq\left.\lambda_{\max }\left(P^{s}\right)\|\varsigma(0)\|^{2}\right|_{s=0} .
$$

Let $\lambda_{2}=\max \left[\lambda_{\min }\left(P^{s}\right)\right], s=1,2,3, \ldots, n_{0}$. Clearly, from inequality (A9), we have

$$
\mathbb{E}\left\{\|\varsigma(k)\|^{2}\right\} \leq\left.\frac{\lambda_{\max }\left(P^{s}\right)}{\lambda_{2}} \sigma_{0}^{k}\|\varsigma(0)\|^{2}\right|_{s=0} .
$$

Hence, the AFES (13) is exponentially stable.

Then, we define

$$
J(k)=\varsigma^{T}(k+1) P(r(k+1)) \varsigma(k+1)-\varsigma^{T}(k) P(r(k)) \varsigma(k) .
$$


Define $\xi(k)=\left[\begin{array}{cc}\varsigma^{T}(k) & \bar{w}^{T}(k)\end{array}\right]^{T}$. In the light of $\tilde{z}^{T}(k) \tilde{z}(k)-\gamma^{2} \bar{w}^{T}(k) \bar{w}(k)-\tilde{z}^{T}(k) \tilde{z}(k)$ $+\gamma^{2} \bar{w}^{T}(k) \bar{w}(k)=0$ and Equation (A11), simultaneously, one has

$$
\mathbb{E}\{J(k)\}=\mathbb{E}\left\{\tilde{\zeta}^{T}(k) \Lambda^{s} \xi(k)-\tilde{z}^{T}(k) \tilde{z}(k)+\gamma^{2} \bar{w}^{T}(k) \bar{w}(k)\right\},
$$

where

$$
\Lambda^{s}=\left[\begin{array}{cc}
\mathscr{A}^{s T} \bar{P}^{s} \mathscr{A}^{s}-P^{s}+\mathscr{M}^{T} \mathscr{M} & * \\
\mathscr{B}^{s T} \bar{P}^{s} \mathscr{A}^{s} & \mathscr{B}^{s T} \bar{P}^{s} \mathscr{B}^{s}-\gamma^{2} I
\end{array}\right] .
$$

Then, by accumulating both sides of Equation (A12) from 0 to $N-1$, we can get

$$
\begin{aligned}
\sum_{k=0}^{N-1} \mathbb{E}\{J(k)\} & =\mathbb{E}\left\{\varsigma^{T}(N) \bar{P}^{s} \varsigma(N)\right\}-\varsigma^{T}(0) P^{s} \varsigma(0) \\
& =\mathbb{E}\left\{\sum_{k=0}^{N-1} \xi^{T}(k) \Lambda^{s} \xi(k)\right\}-\mathbb{E}\left\{\sum_{k=0}^{N-1}\left(\tilde{z}^{T}(k) \tilde{z}(k)-\gamma^{2} \bar{w}^{T}(k) \bar{w}(k)\right)\right\} .
\end{aligned}
$$

Therefore, the $H_{\infty}$ performance condition defined in (16) can be further described as

$$
J=\mathbb{E}\left\{\sum_{k=0}^{N-1} \xi^{T}(k) \Lambda^{s} \xi(k)\right\}-\mathbb{E}\left\{\varsigma^{T}(N) \bar{P}^{s} \varsigma(N)\right\}+\varsigma^{T}(0)\left(P^{s}-\gamma^{2} R^{s}\right) \varsigma(0) .
$$

According to Schur complement lemma, inequality (21) implies $\Lambda^{s} \leq 0$. At the same time, noting $\bar{P}^{s}>0$ and the initial condition $P^{s} \leq \gamma^{2} R^{s}$, we have $J<0$. The proof is completed.

\section{Appendix B. Proof of Theorem 2}

Proof. Construct matrices

$$
G^{s}=\operatorname{diag}\left\{V_{1}^{s}, V_{2}^{s}\right\}, P^{s}=\operatorname{diag}\left\{P_{1}^{s}, P_{2}^{s}\right\}, \bar{P}^{s}=\operatorname{diag}\left\{\bar{P}_{1}^{s}, \bar{P}_{2}^{s}\right\}, \bar{P}_{i}^{s}=\sum_{t=1}^{n_{0}} \pi_{s t} P_{i}^{t},
$$

where $i=1,2, s=1,2, \ldots, n_{0}$.

With the help of Lemma 1, the following inequality can be established based on inequality (21),

$$
\left[\begin{array}{cccc}
-P^{s} & * & * & * \\
0 & -\gamma^{2} I & * & * \\
G^{s} \mathscr{A}^{s} & G^{s} \mathscr{B}^{s} & \bar{P}^{s}-G^{s}-G^{s T} & * \\
\mathscr{M} & 0 & 0 & -I
\end{array}\right]<0
$$

Considering Equations (14) and (A16), we can calculate

$$
\begin{aligned}
& \bar{P}^{s}-G^{s}-G^{s T}=\left[\begin{array}{cc}
\bar{P}_{1}^{s}-V_{1}^{s}-V_{1}^{s T} & * \\
0 & \bar{P}_{2}^{s}-V_{2}^{s}-V_{2}^{s T}
\end{array}\right], \\
& G^{s} \mathscr{A}^{s}=\left[\begin{array}{cc}
V_{1}^{s} \bar{A} & 0 \\
-V_{2}^{s} \bar{L}^{s} \Delta_{q}(k) \bar{C} & V_{2}^{s} \bar{A}-V_{2}^{s} \bar{L}^{s} \bar{C}-V_{2}^{s} \bar{K}^{s} \mathcal{L}^{s}
\end{array}\right], \\
& G^{s} \mathscr{B}^{s}=\left[\begin{array}{cc}
V_{1}^{s} \bar{B} & 0 \\
V_{2}^{s} \bar{B} & -V_{2}^{s} \bar{L}^{s} \bar{D}-V_{2}^{s} \bar{L}^{s} \Delta_{q}(k) \bar{D}
\end{array}\right] .
\end{aligned}
$$


Substituting (A18) into (A17), one has

$$
\left[\begin{array}{ccccccc}
-P_{1}^{s} & * & * & * & * & * & * \\
0 & -P_{2}^{s} & * & * & * & * & * \\
0 & 0 & -\gamma^{2} I & * & * & * & * \\
0 & 0 & 0 & -\gamma^{2} I & * & * & * \\
V_{1}^{s} \bar{A} & 0 & V_{1}^{s} \bar{B} & 0 & \Phi_{55}^{s} & * & * \\
\Phi_{61}^{s} & \Phi_{62}^{s} & V_{2}^{s} \bar{B} & \Phi_{64}^{s} & 0 & \Phi_{66}^{s} & * \\
0 & \bar{M} & 0 & 0 & 0 & 0 & -I
\end{array}\right]<0,
$$

where

$$
\begin{aligned}
& \Phi_{55}^{s}=\bar{P}_{1}^{s}-V_{1}^{s}-V_{1}^{s T}, \Phi_{61}^{s}=-V_{2}^{s} \bar{L}^{s} \Delta_{q}(k) \bar{C}, \\
& \Phi_{62}^{s}=V_{2}^{s} \bar{A}-V_{2}^{s} \bar{L}^{s} \bar{C}-V_{2}^{s} \bar{K}^{s} \mathcal{L}^{s} \\
& \Phi_{64}^{s}=-V_{2} \bar{L}^{s} \bar{D}-V_{2} \bar{L}^{s} \Delta_{q}(k) \bar{D}, \Phi_{66}^{s}=\bar{P}_{2}^{s}-V_{2}^{s}-V_{2}^{s T} .
\end{aligned}
$$

Next, the uncertainty $\Delta_{q}(k)$ introduced by the quantization error is transformed into a norm bounded uncertainty. Considering $k:\left|\Delta_{q_{i}}(k)\right| \leq \delta_{i}, i=1,2, \ldots N$, referring to the technology from the authors of [30], letting $H=\operatorname{diag}\left\{\delta_{1}, \delta_{2}, \ldots, \delta_{N}\right\}$ and $F(k)=H^{-1} \Delta_{q}(k)$, we have

$$
\Delta_{q}(k)=H F(k) .
$$

Inequality (A19) can easily be rewritten as

$$
\begin{gathered}
{\left[\begin{array}{ccccccc}
-P_{1}^{s} & * & * & * & * & * & * \\
0 & -P_{2}^{s} & * & * & * & * & * \\
0 & 0 & -\gamma^{2} I & * & * & * & * \\
0 & 0 & 0 & -\gamma^{2} I & * & * & * \\
V_{1}^{s} \bar{A} & 0 & V_{1}^{s} \bar{B} & 0 & \Phi_{55}^{s} & * & * \\
0 & \Phi_{62}^{s} & V_{2}^{s} \bar{B} & \bar{\Phi}_{64}^{s} & 0 & \Phi_{66}^{s} & * \\
0 & \bar{M} & 0 & 0 & 0 & 0 & -I
\end{array}\right]} \\
+M_{1} F(k) N_{1}+N_{1}^{T} F^{T}(k) N_{1} M_{1}^{T}<0,
\end{gathered}
$$

where

$$
\begin{aligned}
\bar{\Phi}_{64}^{s} & =-V_{2}^{s} \bar{L}^{s} \bar{D}, N_{1}=\left[\begin{array}{llllllll}
\bar{C} & 0 & 0 & \bar{D} & 0 & 0 & 0
\end{array}\right], \\
M_{1} & =\left[\begin{array}{lllllll}
0 & 0 & 0 & 0 & \aleph_{15}^{s T} & \aleph_{16}^{s T} & 0
\end{array}\right]^{T}
\end{aligned}
$$

with $\aleph_{15}^{s}=-H^{T} \bar{L}^{s T} V_{2}^{s T}, \aleph_{16}^{s}=-H^{T} \bar{L}^{s T} V_{2}^{s T}$.

According to Lemma 2, a constant $\varepsilon>0$ can be found, and the following inequality constraint holds

$$
\left[\begin{array}{cccccccc}
\tilde{\Phi}_{11}^{s} & * & * & * & * & * & * & * \\
0 & -P_{2}^{s} & * & * & * & * & * & * \\
0 & 0 & -\gamma^{2} I & * & * & * & * & * \\
\tilde{\Phi}_{41}^{s} & 0 & 0 & \tilde{\Phi}_{44}^{s} & * & * & * & * \\
V_{1}^{s} \bar{A} & 0 & V_{1}^{s} \bar{B} & 0 & \Phi_{55}^{s} & * & * & * \\
0 & \Phi_{62}^{s} & V_{2}^{s} \bar{B} & \bar{\Phi}_{64}^{s} & 0 & \Phi_{66}^{s} & * & * \\
0 & \bar{M} & 0 & 0 & 0 & 0 & -I & * \\
0 & 0 & 0 & 0 & 0 & \tilde{\Phi}_{86}^{s} & 0 & -\varepsilon I
\end{array}\right]<0,
$$

where $\tilde{\Phi}_{11}^{s}=-P_{1}^{s}+\varepsilon^{-1} \bar{C}^{T} \bar{C}, \tilde{\Phi}_{41}^{s}=\varepsilon^{-1} \bar{D}^{T} \bar{C}, \tilde{\Phi}_{44}^{s}=-\gamma^{2} I+\varepsilon^{-1} \bar{D}^{T} \bar{D}, \tilde{\Phi}_{86}^{s}=-\varepsilon H^{T} \bar{L}^{s T} V_{2}^{s T}$

Further, considering that the transition probability of switching topology is not totally available, the transition probability satisfies $\sum_{t=1}^{n_{0}} \pi_{s t}=1$. Therefore, the free-connection 
weighting matrix $W^{s}=\operatorname{diag}\left\{W_{1}^{s}, W_{2}^{s}, \ldots, W_{8}^{s}\right\}>0, s \in \mathcal{S}$ are introduced in the following equation with constant zero,

$$
-\left(\sum_{t \in U_{m}^{s}}^{n_{0}} \pi_{s t}+\sum_{t \in U_{u m}^{s}}^{n_{0}} \pi_{s t}-1\right) W^{s}=0 .
$$

Combining inequalities (A22), (A24) and (A25), it can be deduced that

$$
\begin{gathered}
{\left[\begin{array}{cccccccc}
\Xi_{11}^{s} & * & * & * & * & * & * & * \\
0 & \Xi_{22}^{s} & * & * & * & * & * & * \\
0 & 0 & \Xi_{33}^{s} & * & * & * & * & * \\
\tilde{\Phi}_{41}^{s} & 0 & 0 & \Xi_{44}^{s} & * & * & * & * \\
V_{1}^{s} \bar{A} & 0 & V_{1}^{s} \bar{B} & 0 & \Xi_{55}^{s} & * & * & * \\
0 & \Phi_{62}^{s} & V_{2}^{s} \bar{B} & \bar{\Phi}_{64}^{s} & 0 & \Xi_{66}^{s} & * & * \\
0 & \bar{M} & 0 & 0 & 0 & 0 & \Xi_{77}^{s} & * \\
0 & 0 & 0 & 0 & 0 & \tilde{\Phi}_{86} & 0 & \Xi_{88}^{s}
\end{array}\right]} \\
+\sum_{t \in U_{u m}^{s}}^{n_{0}} \pi_{s t} \operatorname{diag}\left\{-W_{1}^{s},-W_{2}^{s},-W_{3}^{s},-W_{4}^{s}, P_{1}^{t}-W_{5}^{s}, P_{2}^{t}-W_{6}^{s},-W_{7}^{s},-W_{8}^{s}\right\}<0 .
\end{gathered}
$$

In order to obtain the linear matrix inequality constraints that are convenient to solve, the new variables $X^{s}=V_{2}^{s} \bar{K}^{s}$ and $Y^{s}=V_{2}^{s} \bar{L}^{s}$ are introduced into Equation (A26), and Theorem 2 is easily obtained. Now, the proof of Theorem 2 is complete.

\section{References}

1. Rashid, B.; Rehmani, M.H. Applications of Wireless Sensor Networks for Urban Areas: A Survey. J. Netw. Comput. Appl. 2016, 60, 192-219. [CrossRef]

2. Ding, D.; Wang, Z.; Shen, B. Recent Advances on Distributed Filtering for Stochastic Systems over Sensor Networks. Int. J. Gen. Syst. 2014, 43, 372-386. [CrossRef]

3. Ren X.; Shen, L.; Liu, M.; Zhang, X.; Chen, H. Research and Sustainable Design of Wearable Sensor for Clothing Based on Body Area Network. Cogn. Comput. Syst. 2021, 3, 206-220.

4. Zhang, D.; Shi, P.; Zhang, W.A.; Yu, L. Energy-efficient Distributed Filtering in Sensor Networks: A Unified Switched Aystem Approach. IEEE Trans. Cybern. 2017, 47, 1618-1629. [CrossRef]

5. Ren X.; Shen, L.; Zhang, X.; Chen, H.; Huang, Y.; Jin, P. Design and Research of Life-saving Cotton-blended Miners' Clothing. Int. J. Occup. Saf. Ergon. 2021, in press. [CrossRef]

6. Das, S.; Moura, J.M. Consensus+innovations Distributed Kalman Filter with Optimized Gains. IEEE Trans. Signal Process. 2017, 65, 467-481. [CrossRef]

7. Yang, H.; Li, H.; Xia, Y.; Li, L. Distributed Kalman Filtering over Sensor Networks with Transmission Delays. IEEE Trans. Cybern. 2020, in press. [CrossRef] [PubMed]

8. Marelli, D.; Zamani, M.; Fu, M.; Ninness, B. Distributed Kalman Filter in A Network of Linear Systems. Syst. Control Lett. 2018, 116, 71-77. [CrossRef]

9. Li, C.; Dong, H.; Li, J.; Wang, F. Distributed Kalman Filtering for Sensor Network with Balanced Topology. Syst. Control Lett. 2019, 131, 104500. [CrossRef]

10. Rezaei, H.; Mahboobi Esfanjani, R.; Akbari, A.; Sedaaghi, M.H. Event-triggered Distributed Kalman Filter with Consensus on Estimation for State-saturated Systems. Int. J. Robust Nonlinear Control 2020, 30, 8327-8339. [CrossRef]

11. Battistelli, G.; Chisci, L.; Selvi, D. A Distributed Kalman Filter with Event-triggered Communication and Guaranteed Stability. Automatica 2018, 93, 75-82. [CrossRef]

12. Liu, S.; Wang, Z.; Wei, G.; Li, M. Distributed Set-membership Filtering for Multirate Systems Under the Round-robin Scheduling over Sensor Networks. IEEE Trans. Cybern. 2020, 50, 1910-1920. [CrossRef] [PubMed]

13. Shen, B.; Wang, Z.; Wang, D.; Liu, H. Distributed State-saturated Recursive Filtering over Sensor Networks under Round-robin Protocol. IEEE Trans. Cybern. 2020, 50, 3605-3615. [CrossRef] [PubMed]

14. Bu, X.; Dong, H.; Han, F.; Hou, N.; Li, G. Distributed Filtering for Time-varying Systems over Sensor Networks with Randomly Switching Topologies under the Round-robin Protocol. Neurocomputing 2019, 346, 58-64. [CrossRef]

15. Dong, H.; Wang, Z.; Gao, H. Distributed $H_{\infty}$ Filtering for A Class of Markovian Jump Nonlinear Time-delay Systems over Lossy Sensor Networks. IEEE Trans. Ind. Electron. 2013, 60, 4665-4672. [CrossRef]

16. Hu, Z.; Shi, P.; Wu, L.; Ahn, C.K. Event-based Distributed Filtering Approach to Nonlinear Stochastic Systems over Sensor Networks. Int. J. Control. Autom. Syst. 2019, 17, 896-906. [CrossRef] 
17. Shen, B.; Wang, Z.; Liu, X. A Stochastic Sampled-data Approach to Distributed $H_{\infty}$ Filtering in Sensor Networks. IEEE Trans. Circuits Syst. I Regul. Pap. Circuits Syst. I Regul. Pap. 2011, 58, 2237-2246. [CrossRef]

18. Liu, J.; Gu, Y.; Cao, J.; Fei, S. Distributed Event-triggered $H_{\infty}$ Filtering over Sensor Networks with Sensor Saturations and Cyber-attacks. ISA Trans. 2018, 81, 63-75. [CrossRef]

19. Shen, B.; Wang, Z.; Hung, Y.S. Distributed $H_{\infty}$-consensus Filtering in Sensor Networks with Multiple Missing Measurements: The Finite-horizon Case. Automatica, 2010, 46, 1682-1688. [CrossRef]

20. Zhu, F.; Peng, L. Distributed Adaptive Event-triggered Iterative Learning Controller Based on a Filtering Network. Trans. Inst. Meas. Control, 2021, 43, 3220-3232. [CrossRef]

21. Qu, H.; Yang, F. Distributed $H_{\infty}$-consensus Filtering for Target State Tracking over A Wireless Filter Network with Switching Topology, Channel Fading and Packet Dropouts. Neurocomputing 2020, 400, 401-411. [CrossRef]

22. Yan, H.; Yang, Q.; Zhang, H.; Yang, F.; Zhan, X. Distributed $H_{\infty}$ State Estimation for a Class of Filtering Networks with Timevarying Switching Topologies and Packet Losses. IEEE Trans Syst. Man Cybern. Syst. Syst. Man, Cybern. Syst. 2018, 48, $2047-2057$. [CrossRef]

23. Yang, F.; Han, Q.L.; Liu, Y. Distributed $H_{\infty}$ State Estimation over a Filtering Network with Time-varying and Switching Topology and Partial Information Exchange. IEEE Trans. Cybern. 2019, 49, 870-882. [CrossRef]

24. Yu, X.; Lin, Y.; Zhang, X. Global Adaptive Output Feedback Tracking for a Class of Nonlinear Systems with Quantized Input. J. Franklin Inst. 2020, 357, 6083-6095. [CrossRef]

25. Xiang, B.; Chen, B.; Yu, L. Distributed Fusion Estimation for Unstable Systems with Quantized Innovations. IEEE Trans Syst. Man, Cybern. Syst. Syst. Man, Cybern. Syst. 2019, 51, 6381-6387. [CrossRef]

26. Sun, B.; Wen, S.; Wang, S.; Huang, T.; Chen, Y.; Li, P. Quantized Synchronization of Memristive Neural Networks with Timevarying Delays via Super-twisting Algorithm. Neurocomputing 2020, 380, 133-140. [CrossRef]

27. Shao, X.; Shi, Y. Neural Adaptive Control for MEMS Gyroscope with Full-state Constraints and Quantized Input. IEEE Trans. Ind. Informatics 2020, 16, 6444-6454. [CrossRef]

28. Li, Y.; Yang, F. Robust Adaptive Attitude Control for Non-rigid Spacecraft with Quantized Control Input. IEEE/CAA J. Autom. Sin. 2020, 7, 472-481. [CrossRef]

29. Shi, Y.; Shao, X.; Zhang, W. Quantized Learning Control for Flexible Air-breathing Hypersonic Vehicle with Limited Actuator Bandwidth and Prescribed Performance. Aerosp. Sci. Technol. 2020, 97, 105629. [CrossRef]

30. Fu, M.; Xie, L. The Sector Bound Approach to Quantized Feedback Control. IEEE Trans. Automat. Control 2005, 50, 1698-1711.

31. Wang, B.; Cheng, J.; Zhou, X. A Multiple Hierarchical Structure Strategy to Quantized Control of Markovian Switching Systems. Appl. Math. Comput. 2020, 373, 125037. [CrossRef]

32. Sun, K.; Karimi, H.R.; Qiu, J. Finite-time Fuzzy Adaptive Quantized Output Feedback Control of Triangular Structural Systems. Inf. Sci. 2020, 557, 153-169. [CrossRef]

33. Patra, J.C.; Meher, P.K.; Chakraborty, G. Development of Laguerre Neural-network-based Intelligent Sensors for Wireless Sensor Networks. IEEE Trans. Instrum. Meas. 2011, 60, 725-734. [CrossRef]

34. Khachab, N.I.; Mohammed I. Linearization Techniques for $n$ th-order Sensor Models in MOS VLSI Technology. IEEE Trans. Circuits Syst. 1991, 38, 1439-1450. [CrossRef]

35. De Oliveira, M.C.; Bernussou, J.; Geromel, J.C. A New Discrete-time Robust Stability Condition. Syst. Control Lett. 1999, 37, 261-265. [CrossRef]

36. Yang, W.; Liu, M.; Shi, P. $H_{\infty}$ Filtering for Nonlinear Stochastic Systems with Sensor Saturation, Quantization and Random Packet Losses. Signal Process. 2012, 92, 1387-1396. [CrossRef] 